\title{
Empathy, burnout, life satisfaction, correlations and associated socio- demographic factors among Chinese undergraduate medical students: an exploratory cross-sectional study
}

\author{
Qinghua Wang ${ }^{1}$, Lie Wang ${ }^{2}$, Meng Shi ${ }^{1}$, Xuelian $\mathrm{Li}^{3}$, Rong Liư ${ }^{4}$, Jie Liư ${ }^{4}$, Min Zhu ${ }^{5}$ and Huazhang $\mathrm{Wu}^{5^{*}}$
}

\begin{abstract}
Background: Medical education is widely known to be a demanding process that may cause various mental health problems, such as burnout, which can lead to lowered levels of life satisfaction among medical students. Research shows that empathy is negatively correlated with burnout, but there are few studies on the relationship among empathy, burnout and life satisfaction in medical students. The objective of the present study is to explore the correlations of empathy and burnout with life satisfaction and the associated socio-demographic factors among Chinese undergraduate medical students.
\end{abstract}

Methods: In this cross-sectional study, 1271 undergraduate medical students (age $19.42 \pm 1.34$ years, 36\% male) from 1st to 4th grades completed questionnaires including the Interpersonal Reactivity Index Chinese version (IRIC), the Maslach Burnout Inventory Modified Chinese version (MBI-MC), the Satisfaction With Life Scale (SWLS) and socio-demographic characteristics. Statistical analyses included Student's t-test, one-way ANOVA, post hoc Bonferroni tests, hierarchical linear regression analysis and general linear model-univariate full factorial model.

Results: Over four academic years, medical students' empathy levels declined, but their burnout levels almost plateaued and their life satisfaction levels witnessed an initial fall before a rebound. Empathy was correlated with students' age and grade, and burnout was associated with students' maternal education. Significant differences in life satisfaction were detected with regard to medical students' age, academic year, the number of children in the family, place of residence and parents' educational levels.

Conclusions: Empathy explained $0.6 \%$ of the variance in life satisfaction in contrast to $13.7 \%$ of the variance explained by burnout in life satisfaction. Although empathy did not have a main effect on life satisfaction, there was an interaction effect of empathy and burnout on life satisfaction among students of high and low empathy and burnout levels. Students with high levels of empathy and low levels of burnout were most satisfied with life. Medical institutions and related authorities need to find effective measures to enhance students' empathy levels and reduce burnout to improve their life satisfaction.

Keywords: Empathy, Burnout, Life satisfaction, Medical students

\footnotetext{
* Correspondence: wuhuazhang@126.com

${ }^{5}$ Department of Healthcare Management, School of Humanities and Social

Sciences, China Medical University, No. 77 Puhe Road, Shenyang North New

Area, Shenyang, Liaoning Province, People's Republic of China

Full list of author information is available at the end of the article
}

(c) The Author(s). 2019 Open Access This article is distributed under the terms of the Creative Commons Attribution 4.0 International License (http://creativecommons.org/licenses/by/4.0/), which permits unrestricted use, distribution, and reproduction in any medium, provided you give appropriate credit to the original author(s) and the source, provide a link to the Creative Commons license, and indicate if changes were made. The Creative Commons Public Domain Dedication waiver (http://creativecommons.org/publicdomain/zero/1.0/) applies to the data made available in this article, unless otherwise stated. 


\section{Background}

It is widely acknowledged that medical professionals are subjected to heavy workloads, long work hours, a stressful work environment, high job pressure, and an intense physician-patient relationship, which lead to various mental health problems, such as anxiety, depression and burnout. These psychological problems exert detrimental effects on the life quality and life satisfaction of health professionals, which in turn impact the quality of care they provide to patients $[1,2]$.

One often cited factor related to occupational burnout among medical professionals is compassion fatigue, which is closely associated with empathy [3, 4]. Empathy refers to the ability to stand in others' shoes, to understand as well as to share other people's emotions and feelings and the ability to communicate this understanding [5]. Empathy is an essential element in the physician-patient relationship and affects clinical outcomes, patient compliance and satisfaction $[6,7]$. Therefore, great importance is attached to empathy in undergraduate medical education, the critical phase in nurturing competent physicians, and the development of empathy in medical students is considered a goal in medical education [8].

The literature indicates that occupational burnout may have its root in the medical schools where health professionals were trained as students. In addition to stress, anxiety and depression, burnout is known to be another mental health problem prevalent in medical students. Burnout is defined as a syndrome of prolonged emotional exhaustion, depersonalization (cynicism) and a feeling of professional inefficacy [9]; possible reasons that medical students are prone to burnout problems are the intensive medical curriculum, lack of social support and inappropriate coping styles. Studies show that burnout can occur from the outset of undergraduate medical education during preclinical years [10], and the problem becomes more serious with the increase of grade levels [11].

The negative association of empathy with burnout among medical professionals is recorded in a large number of studies [1-4, 12-15]. Regarding the correlation of empathy and burnout among medical students, crosssectional multi-institutional research conducted in Brazil concluded that personal accomplishment, one facet of the Maslach Burnout Inventory (MBI), held the strongest negative association with personal distress, which is a subscale of the Interpersonal Reactivity Index (IRI) [16]. Another study with a sample of 265 third-year medical students found that empathy measured by the Jefferson Scale of Empathy (JSE) was positively correlated with personal accomplishment while negatively associated with depersonalization, which are two subscales of the MBI [17]. Lucchetti and colleagues conducted cross-cultural empirical research comparing the differences between
American and Brazilian medical students in terms of mental health, life quality, empathy and burnout. The results indicated that US medical students scored significantly higher in empathy as measured by the Empathy, Spirituality, and Wellness in Medicine survey (ESWIM) than their Brazilian counterparts whereas American medical students were more likely to suffer from exhaustion as measured by the Oldenburg Burnout Inventory (OLBI) [18].

Studies consistently showed that burnout is significantly negatively related to life satisfaction among medical students and health professionals. Life satisfaction, as a concept concerning the cognitive aspect of subjective wellbeing, is defined as an individual's conscious evaluation of his or her life based on self-set standards [19]. The research conducted among 452 pharmacy students from five South Korean universities using structural equation modelling (SEM) found that exhaustion and cynicism, which are two facets of the Maslach Burnout Inventory-Student Survey (MBI-SS), were both significantly negatively correlated with life satisfaction [20]. A three-wave seven-year prospective study among 3255 Finnish dentists revealed that burnout predicted depressive symptoms and lowered life satisfaction [21]. Demerouti and colleagues detected a significantly negative relationship between exhaustion and disengagement, which are two facets of the Oldenburg Burnout Inventory (OLBI), and life satisfaction among 109 German nurses [22]. Although previous studies have confirmed the associations among empathy, burnout and life satisfaction, to the best of our knowledge, there has been no study exploring the possible interaction effect of empathy and burnout on life satisfaction in medical students. Additionally, whereas some previous studies have examined the associated socio-demographic factors such as age, gender and grade with empathy, burnout and life satisfaction, no research has examined the correlations of socio-demographic factors such as place of residence and parents' educational levels with empathy, burnout and life satisfaction simultaneously in Chinese undergraduate medical students. Therefore, the aims of the present study were to (i) investigate the associated sociodemographic factors with empathy, burnout and life satisfaction and (ii) determine whether there is an interaction effect of empathy and burnout on life satisfaction in Chinese undergraduate medical students.

\section{Methods}

\section{Study design and subjects}

This cross-sectional study was conducted at China Medical University, which is a major medical university in Northeast China. A stratified random cluster sampling method was adopted, and questionnaires were distributed from September to November 2018 to whole classes of medical students in their 1st to 4th academic year 
of studies. The purpose of the survey was explained beforehand, and participation was voluntary. Written informed consent was obtained from every subject, and the study was approved by the China Medical University Committee on Human Experimentation.

\section{Measurement of empathy}

The two most widely used measurement scales of empathy are the Jefferson Scale of Empathy (JSE) [23] and the Interpersonal Reactivity Index (IRI) [24]. Considering the large number of preclinical student participants who had little or no contact with real patients in this study, we used the IRI to measure empathy. With permission from the author of the IRI Mark H. Davis, Zhan [25], a scholar from Taiwan, translated the IRI scale bidirectionally and tested the validity as well as reliability of the IRI Chinese version and adapted the original 28item scale into the 22-item IRI scale Chinese version (IRI-C). The IRI-C included four subscales: Perspective Taking (PT: 5 items), Personal Distress (PD: 5 items), Fantasy Scale (FS: 6 items) and Empathic Concern (EC: 6 items). Each item was scored on a 5-point Likert scale from 0 (not true of me at all) to 4 (very true of me). Rong and colleagues [26] tested the IRI-C in Chinese college students and demonstrated the sound psychometric properties of the scale. The Cronbach's alpha coefficients for the four subscales in the present study were PT: 0.767 , PD: 0.806 , FS: 0.665 , EC: 0.615 and the Cronbach's alpha coefficient for the total scale was 0.760 .

\section{Measurement of burnout}

The Maslach Burnout Inventory Modified Chinese version (MBI-MC) was adapted by Lian [27], which was the most widely used burnout inventory among college students in China. Based on the MBI, the MBI-MC reduced the 22 items in the original scale to a 20-item scale that contained three subscales: Low Mood (LM: 8 items), Improper Behaviour (IB: 6 items) and Low Achievement (LA: 6 items). Example questions in the MBI-MC are "I feel exhausted after studying for a whole day" and "I can calmly handle my emotional problems". Each item was scored on a 5-point Likert scale from 1 (not true of me at all) to 5 (very true of me), and after negatively worded questions were reverse scored, the sum score was calculated to measure medical students' overall level of burnout. The Cronbach's alpha coefficients for the three subscales in the present study were LM: 0.828, IB: 0.702, LA: 0.664 and the Cronbach's alpha coefficient for the total scale was 0.872 .

\section{Measurement of life satisfaction}

Medical students' levels of life satisfaction were measured by the Satisfaction With Life Scale (SWLS) [19], which consisted of 5 items scored on a 7-point Likert scale. Each item received a score ranging from 1 (strongly disagree) to 7 (strongly agree), and the total score was calculated to indicate the students' overall level of life satisfaction. The Chinese version of the scale demonstrated good validity and reliability among medical students in previous studies [28, 29], and the Cronbach's alpha coefficient for the scale in the present study was 0.882 .

\section{Demographic characteristics}

Demographic information included participants' gender, age, grade, race, the number of children in the family, place of residence and parents' educational levels. Students were categorized into two age groups: 17-19 and 20-24 years old. With regard to Chinese culture, race was divided into five groups: Han, Hui, Man, Wei and Other. The number of children in the family was dichotomized into only child or not. Place of residence fell into three categories: cities, towns, and villages. Parents' education was divided into three levels: primary school and below, secondary school, college and above.

\section{Statistical analysis}

Student's t-test and one-way ANOVA were used to examine the differences in empathy, burnout and life satisfaction within the categorical demographic variables. Post hoc Bonferroni tests were performed to examine if the difference was significant between every two groups concerning continuous variables with regard to multicategorical demographic variables. Hierarchical linear regression analysis was utilized to explore the association of empathy and burnout with life satisfaction. General linear model-univariate full factorial model was used to calculate main effects, the interaction effect and simple effects of two independent variables of empathy and burnout on the dependent variable of life satisfaction. All the statistical tests were performed using SPSS 22.0 with the significance level set at $p<0.05$ (two-tailed).

\section{Results \\ Demographic characteristics and differences in empathy, burnout and life satisfaction}

The differences in the continuous variables of empathy, burnout and life satisfaction within the sociodemographic categorical variables of the subjects are presented in Table 1. Of the 1563 invited medical students, 1271 agreed to participate, signed the written informed consent forms and completed the questionnaires, with a response rate of $81.3 \%$. The average age of the participants was $19.42 \pm 1.34$ years old, and the number of males was 458 (36\%), while the number of females was $813(64 \%)$. As can be seen from Table 1, the empathy level of students aged 17-19 was significantly higher than that of students aged $20-24$ ( 52.78 vs $51.10, p=0.005$ ), and students of various grades saw a significant difference in terms of their 
Table 1 Demographic characteristics and differences in empathy, burnout and life satisfaction $(N=1271)$

\begin{tabular}{|c|c|c|c|c|c|c|c|}
\hline Variables & N (\%) & $\mid \mathrm{RI}-\mathrm{C}(\mathrm{M} \pm \mathrm{SD})$ & $p$ & MBI-MC $(\mathrm{M} \pm \mathrm{SD})$ & $p$ & $\operatorname{SWLS}(M \pm S D)$ & $p$ \\
\hline Gender & & & 0.116 & & 0.091 & & 0.052 \\
\hline Male & $458(36.0)$ & $51.44 \pm 10.02$ & & $53.30 \pm 12.18$ & & $22.14 \pm 6.78$ & \\
\hline Female & $813(64.0)$ & $52.40 \pm 10.70$ & & $52.13 \pm 11.63$ & & $22.88 \pm 6.44$ & \\
\hline Age & & & 0.005 & & 0.697 & & 0.018 \\
\hline $17-19$ & 725 (57.0) & $52.78 \pm 10.60$ & & $52.66 \pm 11.55$ & & $22.99 \pm 6.27$ & \\
\hline $20-24$ & $546(43.0)$ & $51.10 \pm 10.23$ & & $52.40 \pm 12.21$ & & $22.11 \pm 6.93$ & \\
\hline Academic year & & & 0.001 & & 0.751 & & 0.028 \\
\hline First year & $415(32.7)$ & $53.66 \pm 10.12$ & & $52.33 \pm 10.92$ & & $23.33 \pm 6.20$ & \\
\hline Second year & 341 (26.8) & $51.73 \pm 10.91$ & & $53.15 \pm 12.98$ & & $22.26 \pm 6.43$ & \\
\hline Third year & $264(20.8)$ & $51.83 \pm 10.41$ & & $52.30 \pm 11.25$ & & $21.91 \pm 6.78$ & \\
\hline Fourth year & $251(19.7)$ & $50.10 \pm 10.15$ & & $52.35 \pm 12.31$ & & $22.67 \pm 7.05$ & \\
\hline Race & & & 0.417 & & 0.979 & & 0.044 \\
\hline Han & 1030(81.0) & $52.00 \pm 10.62$ & & $52.56 \pm 12.05$ & & $22.50 \pm 6.64$ & \\
\hline Hui & $18(1.4)$ & $48.06 \pm 8.71$ & & $51.22 \pm 9.16$ & & $24.00 \pm 5.58$ & \\
\hline Man & $108(8.5)$ & $52.17 \pm 9.66$ & & $52.28 \pm 11.53$ & & $24.13 \pm 6.29$ & \\
\hline Wei & $29(2.3)$ & $52.69 \pm 7.76$ & & $52.52 \pm 8.44$ & & $23.45 \pm 5.58$ & \\
\hline Other & $86(6.8)$ & $53.26 \pm 10.69$ & & $53.06 \pm 11.30$ & & $21.50 \pm 6.41$ & \\
\hline The only child & & & 0.078 & & 0.302 & & $<0.001$ \\
\hline Yes & 745 (58.6) & $51.62 \pm 10.40$ & & $52.26 \pm 11.95$ & & $23.28 \pm 6.56$ & \\
\hline No & $526(41.4)$ & $52.67 \pm 10.54$ & & $52.96 \pm 11.67$ & & $21.67 \pm 6.48$ & \\
\hline Place of residence & & & 0.214 & & 0.209 & & $<0.001$ \\
\hline Cities & $609(47.9)$ & $52.08 \pm 10.58$ & & $51.98 \pm 12.45$ & & $23.53 \pm 6.49$ & \\
\hline Towns & $313(24.6)$ & $51.28 \pm 10.53$ & & $52.77 \pm 11.35$ & & $22.50 \pm 6.77$ & \\
\hline Villages & $349(27.5)$ & $52.71 \pm 10.20$ & & $53.35 \pm 11.13$ & & $21.12 \pm 6.27$ & \\
\hline Paternal education & & & 0.789 & & 0.334 & & $<0.001$ \\
\hline Primary school and below & $133(10.4)$ & $52.60 \pm 10.65$ & & $53.95 \pm 11.53$ & & $20.48 \pm 6.65$ & \\
\hline Secondary school & $719(56.6)$ & $51.93 \pm 10.58$ & & $52.48 \pm 11.69$ & & $22.47 \pm 6.36$ & \\
\hline College and above & 419 (33.0) & $52.10 \pm 10.24$ & & $52.22 \pm 12.18$ & & $23.54 \pm 6.75$ & \\
\hline Maternal education & & & 0.099 & & 0.005 & & $<0.001$ \\
\hline Primary school and below & $187(14.7)$ & $53.57 \pm 10.40$ & & $54.77 \pm 10.83$ & & $20.46 \pm 6.32$ & \\
\hline Secondary school & $706(55.6)$ & $51.75 \pm 10.55$ & & $52.61 \pm 11.71$ & & $22.55 \pm 6.39$ & \\
\hline College and above & $378(29.7)$ & $51.87 \pm 10.30$ & & $51.32 \pm 12.40$ & & $23.80 \pm 6.77$ & \\
\hline Total sample & $1271(100.0)$ & $52.06 \pm 10.47$ & & $52.55 \pm 11.84$ & & $22.62 \pm 6.57$ & \\
\hline
\end{tabular}

Significance level: $p<0.05$ (two-tailed); M \pm SD: mean \pm standard deviation; IRI-C Interpersonal Reactivity Index Chinese version, MBI-MC Maslach Burnout Inventory Modified Chinese version, SWLS Satisfaction With Life Scale

IRI-C scores $(p<0.001)$. Burnout levels were associated with medical students' maternal education $(p=0.005)$. Regarding life satisfaction, students aged 17-19 were more satisfied with life than those aged $20-24$ (22.99 vs 22.11, $p=0.018$ ), and students who were the only child in the family saw higher levels of life satisfaction (23.28 vs 21.67 , $p<0.001)$. Significant differences were also observed in life satisfaction with regard to medical students' academic year $(p=0.028)$, race $(p=0.044)$, place of residence $(p<0.001)$, paternal education $(p<0.001)$ and maternal education $(p<0.001)$.
Significant differences in empathy, burnout and life satisfaction within socio-demographic multicategorical variables

The significant differences in empathy, burnout and life satisfaction with regard to socio-demographic multicategorical variables were examined by ANOVA post hoc Bonferroni tests. Before the tests, the trial on equality of variance was performed to guarantee that equal variance was achieved, and the results of Bonferroni tests show that the empathy level of 1st-year medical students was significantly higher than that of $4^{\text {th }}$-year students (53.66 
vs $50.10, p<0.001)$. For burnout levels, students whose mothers held college and above qualifications were less likely to suffer from burnout problems than students whose mothers' educational level was primary school and below (51.32 vs $54.77, p=0.003)$. In terms of life satisfaction, 1st-year medical students enjoyed life more than their $3^{\text {rd }}$-year counterparts $(23.33$ vs $21.91, p=$ 0.035). Though one-way ANOVA shows that students of different ethnic groups saw different levels of life satisfaction $(p=0.044)$, no significant differences were detected by post hoc Bonferroni tests. Regarding place of residence, students from cities and towns saw higher life satisfaction levels than those from villages $\left(\mathrm{M}_{\text {cities }}=\right.$ 23.53 vs $\mathrm{M}_{\text {villages }}=21.12, p<0.001 ; \quad \mathrm{M}_{\text {towns }}=22.50$ vs $\left.\mathrm{M}_{\mathrm{villages}}=21.12, p=0.020\right)$. Both maternal and paternal educational levels were correlated with medical students' life satisfaction, with students whose parents held college and above qualifications enjoying life most ( $\mathrm{M}_{\text {paternal edu- }}$ cation (college and above) $=23.54$ vs $M_{\text {paternal education (secondary }}$ school) $=22.47, \quad p=0.023 ; M_{\text {paternal education (college and }}$ above) $=23.54$ vs $M_{\text {paternal education (primary school and below) }}=$

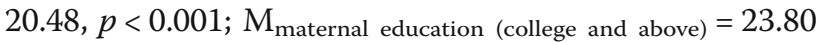
vs $M_{\text {maternal education (secondary school) }}=22.55, p=0.008$; $M_{\text {maternal education (college and above) }}=23.80$ vs $M_{\text {maternal edu- }}$ cation (primary school and below) $=20.46, p<0.001$ ). Students whose parents held secondary school qualifications enjoyed higher life satisfaction levels than those whose parents' educational levels were primary school and below $\left(\mathrm{M}_{\text {paternal education (secondary school) }}=22.47\right.$ vs $\mathrm{M}_{\text {pater- }}$ nal education (primary school and below) $=20.48, p=0.004 ; \mathrm{M}_{\text {ma- }}$ ternal education (secondary school) $=22.55 \mathrm{vs} \mathrm{M}_{\text {maternal education }}$ (primary school and below) $=20.46, p<0.001)$.

The trend in mean scores of empathy, burnout and life satisfaction of undergraduate medical students over four academic years

Figure 1 shows the trend of the $1^{\text {st }}$ to 4 th-year medical students' mean scores in empathy, burnout and life satisfaction. As revealed in the figure, there was an overall downward trend in empathy, with a significant difference detected between the 1st and $4^{\text {th }}$ grades (53.66 vs $50.10, p<0.001)$. As for burnout, there was almost a leveling out since no significant differences were observed between students of various academic years. Life satisfaction saw another pattern, with the mean scores showing an initial fall to the lowest in $3^{\text {rd }}$-year students before rising in $4^{\text {th }}$-year students to a level comparable to that of $1^{\text {st }}$-year students. Overall, $1^{\text {st }}$-year medical students enjoyed life most and their mean score in life satisfaction was significantly higher than the score of $3^{\text {rd }}$-year students $(23.33$ vs $21.91, p<0.05)$.

\section{Results of hierarchical linear regression analysis}

Hierarchical linear regression analysis was adopted to explore the correlation of empathy and burnout with life satisfaction and the results were presented in Table 2 . As can be seen from the table, demographic factors of age and gender explained $0.9 \%$ of the variance in life satisfaction. After empathy was added in step 2, the $\mathrm{F}$ value for the model was still significant $(\mathrm{F}=6.287, p<0.001)$ with empathy significantly positively predicting life satisfaction $(\beta=0.075, p<0.01)$, which accounted for another $0.6 \%$ of the variance in life satisfaction. The association between burnout and life satisfaction was significantly negative $(\beta=-0.371, p<0.001)$, explaining additional $13.7 \%$ of the variance in life satisfaction. It is noticeable that in all three models age was significantly negatively associated with life satisfaction $(\beta=-0.078, p<0.01$ in step $1, \beta=-0.071, p<0.05$ in step 2 and $\beta=-0.074$, $p<0.01$ in step 3 , respectively).

\section{Main effects and the interaction effect of empathy and burnout on life satisfaction}

General Linear Model-Univariate Full Factorial Model was adopted to examine the main effects and to explore whether there was an interaction effect of empathy and
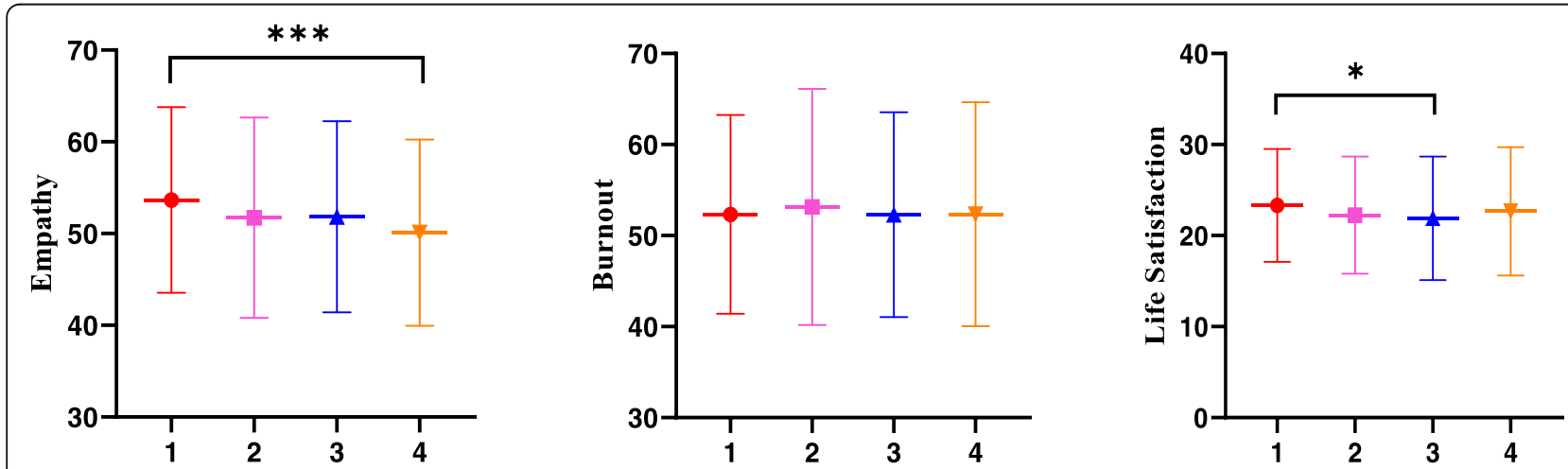

Fig. 1 The trend in mean scores of empathy, burnout and life satisfaction of undergraduate medical students over a span of four academic years (presented as mean \pm standard deviation). Total sample: $N=1271$; 1: first year students $(n=415)$; 2 second year students $(n=341) ; 3$ : third year students $(n=264)$; 4 : fourth year students $(n=251) .{ }^{*} p<0.05 ;{ }^{* * *} p<0.001$ 
Table 2 Hierarchical linear regression analysis with life satisfaction as the dependent variable

\begin{tabular}{|c|c|c|c|}
\hline Variables & Step 1 ( $\beta$ ) & Step $2(\beta)$ & Step $3(\beta)$ \\
\hline \multicolumn{4}{|l|}{ Step 1} \\
\hline Age & $-0.078^{* *}$ & $-0.071^{*}$ & $-0.074^{* *}$ \\
\hline Gender & 0.053 & 0.050 & 0.033 \\
\hline \multicolumn{4}{|l|}{ Step 2} \\
\hline Empathy & & $0.075^{* *}$ & $0.053^{*}$ \\
\hline \multicolumn{4}{|l|}{ Step 3} \\
\hline Burnout & & & $-0.371^{* * *}$ \\
\hline $\mathrm{F}$ & $5.833^{* *}$ & $6.287^{* * *}$ & $56.592^{* * *}$ \\
\hline$R^{2}$ & 0.009 & 0.015 & 0.152 \\
\hline$\Delta R^{2}$ & 0.009 & 0.006 & 0.137 \\
\hline
\end{tabular}

burnout on life satisfaction. We adopted two factors with two levels of factorial design in our study, and since there was no standard for cut-off points, we used the lower and upper quartiles to dichotomize the levels of empathy and burnout into low $(<25 \%)$ and high $(>75 \%)$. Before the test, homogeneity of variance was checked by one-way ANOVA, and the result shows that Levene Statistic $=0.959, p=0.412$, so homogeneity of variances was achieved. Table 3 demonstrates the results of betweensubjects effects tests, and as revealed in the table, burnout had a main effect on medical students' life satisfaction $\left(F(1,351)=87.008, p<0.001, \eta_{\mathrm{p}}{ }^{2}=0.199\right)$. Post hoc Bonferroni tests of the main effect revealed that life satisfaction levels of students with low burnout were significantly higher than those with high burnout levels $\left(\mathrm{M}_{\text {life satisfaction (low burnout) }}=25.66 \mathrm{vs} \mathrm{M}_{\text {life satisfaction (high }}\right.$ burnout) $=19.39, p<0.001$ ) (for details, please refer to the Additional file 1). Whereas the main effect of empathy on life satisfaction did not reach significance level $(F(1$, $\left.351)=0.362, p=0.548, \eta_{\mathrm{p}}{ }^{2}=0.001\right)$, there was an interaction effect of empathy and burnout on life satisfaction $\left(F(1,351)=8.195, \quad p=0.004, \quad \eta_{\mathrm{p}}{ }^{2}=0.023\right)$. The whole model explained $21.3 \%$ of the variance in life satisfaction (adjusted $\mathrm{R}^{2}=0.213$ ).

\section{Simple effects of two levels of empathy and burnout on life satisfaction}

Post hoc Bonferroni tests were performed to explore the simple effects of two levels of empathy and burnout on life satisfaction. The results of pairwise comparisons (for details, please refer to the Additional file 1) show that at the low burnout level, the mean difference in life satisfaction between students of low empathy and high empathy was significant $(24.49$ vs $26.82, F(1,351)=5.970$, $p=0.015, \eta_{\mathrm{p}}^{2}=0.017$ ), while at the high burnout level, the mean difference in life satisfaction between students of low empathy and high empathy was nonsignificant $\left(20.15\right.$ vs $\left.18.63, F(1,351)=2.569, p=0.110, \eta_{\mathrm{p}}{ }^{2}=0.007\right)$. When the level of empathy was fixed at low, the mean difference in life satisfaction between students of low burnout and high burnout was significant (24.49 vs $\left.20.15, F(1,351)=20.705, p<0.001, \eta_{\mathrm{p}}{ }^{2}=0.056\right)$. It is noticeable that when the level of empathy was fixed at high, there was a statistically significant difference with a larger effect size in the mean value of life satisfaction between low burnout and high burnout students (26.82 vs 18.63, $\left.F(1,351)=75.008, p<0.001, \eta_{\mathrm{p}}^{2}=0.176\right)$. Figure 2 shows the simple effects of two levels of empathy and burnout on life satisfaction by post hoc Bonferroni tests.

\section{Discussion}

This is the first study examining the associated sociodemographic factors with empathy, burnout and life satisfaction and the possible interaction effect of empathy and burnout on life satisfaction in Chinese undergraduate medical students. As for the correlation of empathy with socio-demographic characteristics, it is found that younger and lower-grade students were more empathetic than their older higher-grade counterparts. This finding is consistent with the conclusion in a study by $\mathrm{Li}$ and colleagues [30], but in contradiction with the result of another study by Wen [31]. It is worth mentioning that

Table 3 Main effects and the interaction effect of empathy and burnout on life satisfaction

\begin{tabular}{|c|c|c|c|c|c|c|}
\hline Source & SS & df & MS & $\mathrm{F}$ & $p$ & $n p^{2}$ \\
\hline Corrected Model & $3893.306^{a}$ & 3 & 1297.769 & 32.906 & $<0.001$ & 0.220 \\
\hline Intercept & $177,449.242$ & 1 & $177,449.242$ & 4499.334 & $<0.001$ & 0.928 \\
\hline Empathy & 14.268 & 1 & 14.268 & 0.362 & 0.548 & 0.001 \\
\hline Burnout & 3431.491 & 1 & 3431.491 & 87.008 & $<0.001$ & 0.199 \\
\hline Empathy * Burnout & 323.191 & 1 & 323.191 & 8.195 & 0.004 & 0.023 \\
\hline Error & $13,843.088$ & 351 & 39.439 & & & \\
\hline Total & $199,599.000$ & 355 & & & & \\
\hline Corrected Total & $17,736.394$ & 354 & & & & \\
\hline
\end{tabular}

${ }^{\mathrm{a}} \mathrm{R}$ Squared $=.220$ (Adjusted R Squared $=.213$ ); Dependent variable: life satisfaction; SS type III sum of squares, $d f$ degree of freedom, $M S$ mean square, $\eta_{p}{ }^{2}$ partial eta squared. Effect size was measured by partial eta squared: small $=0.01$ to 0.06 , medium $=0.06$ to 0.138 , large $>0.138$ 


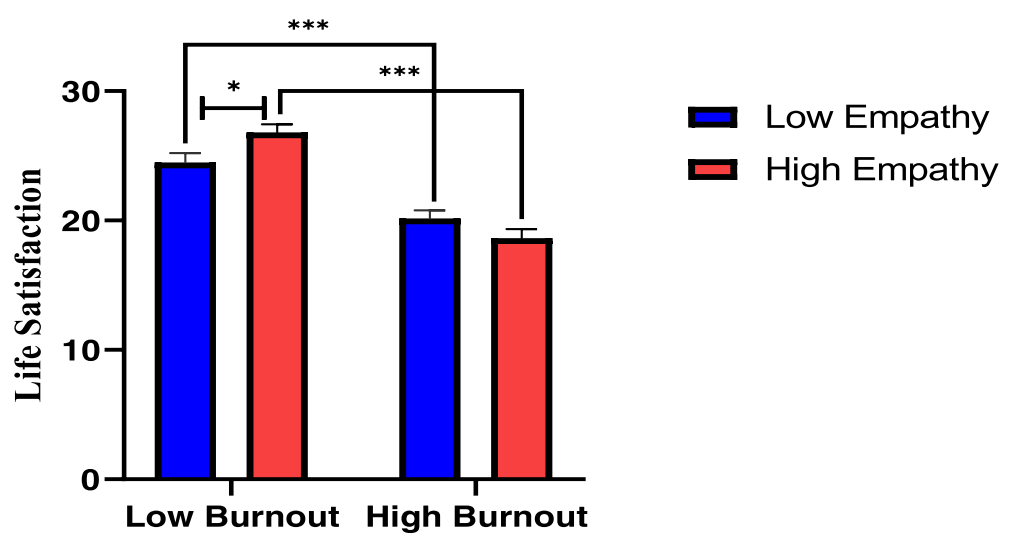

Fig. 2 Simple effects of two levels of empathy and burnout on life satisfaction

although the sample in these two studies was Chinese medical students, the scale used for measuring empathy was the student version of the Jefferson Scale of Physician Empathy (JSPE-S). Research results concerning empathy in medical students from other cultures were also inconsistent. A study with a sample of 320 medical students across the $1^{\text {st }}$ to 6 th academic years in Brazil detected no significant differences in empathy considering students' grade [32], whereas research in Iran indicated that the overall level of empathy among medical students in the preclinical period was higher than that in the clinical period [33]. However, another study from India showed that medical students' empathy scores had a tendency to decline initially and then rebound over time [34]. The contradiction in these findings may be due to the differences in measurement tools, cultures and the environment in which the empirical research was carried out. Another point worth mentioning is that no significant difference in the mean empathy score was found between males and females in the present study, which was in line with the conclusion of the study by $\mathrm{Li}$ [30], although a number of other studies revealed that female medical students were more empathetic than males [31-34].

A new finding in the present study was that medical students' burnout levels were related to maternal but not paternal educational levels, and students whose mothers held college and above qualifications were less likely to suffer from burnout problems than students whose mothers' educational level was primary school and below. In China, as in most other countries around the world, mothers still play a major role in children's growth and education and spend relatively more time with children in comparison to fathers. Research shows that maternal education is inextricably correlated with children's language skills, academic performance, intellectual functioning and maladaptive behaviours [35-37]. Therefore, we assume that more adaptive coping strategies were likely to be adopted by medical students whose mothers were of higher educational levels when they encountered difficulties and felt exhausted. This problem-based coping strategy, along with adjusted behaviours may help students recover from academic and emotional fatigue; thus, they experienced less burnout. The finding that no significant difference was detected in the burnout levels of students in different academic years was in line with the research conducted in 22 medical schools in Brazil [16], which concluded that burnout problems existed in all stages of medical education.

Regarding life satisfaction, students of the 17-19 age group enjoyed life more than those in the 20-24 age group. This finding is inconsistent with the conclusion in Shi [29], which did not show significant differences in the life satisfaction levels of Chinese medical students in different age groups. It is noteworthy that students in the 17-19 age group also had higher levels of empathy in the present study, and since empathy was found to be negatively correlated with stress and burnout but positively associated with social support $[16,38]$, it is reasonable that students with higher levels of empathy also enjoyed higher levels of life satisfaction. In the current study, $1^{\text {st }}$-year medical students had significantly higher life satisfaction scores than $3^{\text {rd }}$-year students but not 4th -year students, which can be explained from the perspective that the 3rd academic year in China is the period in which undergraduate medical students transitioned from preclinical to clinical studies, and this critical transitional period may bring maladjustment and mental health problems such as stress, anxiety and depression that lead to decreased life satisfaction. In the 4th academic year, medical students gradually adapt to the curriculum and learning environment, such as internships in university-affiliated hospitals, so the levels of life satisfaction rebound. As expected, students who were the only child in the family, who were from cities and towns and whose parents were of higher educational 
levels enjoyed higher levels of life satisfaction since they were more likely to have better living conditions and receive better education. It is worth mentioning that the difference in life satisfaction was marginally significant with regard to gender in this study, with females more satisfied with life than their male counterparts (22.88 vs 22.14, $p=0.052$ ). This finding can be explained by the one-child policy in effect in China for several decades; under this policy, boys were more valued and parental expectations for them were higher, and compared with females, males shouldered more pressure and responsibilities, as they were considered pillars of the family, so they were more likely to suffer from mental health problems such as depression, which was demonstrated in some studies of Chinese medical students [39, 40], and this may lead to lower life satisfaction in males.

Hierarchical linear regression analysis shows that burnout explained a large proportion of the variance $(13.7 \%)$ in life satisfaction, whereas empathy only accounted for $0.6 \%$ of the variance in life satisfaction. Age was found to significantly negatively predict life satisfaction and this result was in line with the finding in the present study that students of the 17-19 age group were more satisfied with life than those in the 20-24 age group. With the increase of age and grade, medical students need to deal with more academic work, especially when they started their clinical phase study. The maladjustment brought by the transitional period from preclinical to clinical learning environment and the heavy workload may result in decreased life satisfaction levels.

The results of ANOVA show that burnout had a main effect on life satisfaction, and to effectively address burnout problems, we need to find stressors that lead to burnout in medical students. First, the intensive curriculum and the rigid scoring system in medical education were found to be a source of stress. Slavin and colleagues [41] instituted curricular changes including a pass/fail grading system and reduced contact hours for preclinical students and found that there was a significant decline in medical students' stress, anxiety and depression levels. Second, learning environments such as clinical rotation factors for clinical students and personal characteristics and life events were significant contributors to burnout. A study by Dyrbye [42] detected the associations of call frequency and clinical workload in rotation with burnout among medical students in their clinical practice environment, and the study also concluded that personal life events, gender and learning environment were all factors associated with burnout among preclinical students. Third, lack of social support and low empathy levels as well as dysfunctional coping strategies were detected in some studies as risk factors for burnout [43, 44]. It is worth mentioning that, in the research by Mazurkiewicz and colleagues, control over daily schedule and one's self-efficacy were also found to be related to medical students' burnout [10]. In addition to reforming the medical curriculum and scoring system, improving the learning environment, promoting social support and functional coping strategies, nurturing students' resilience and empathy were all found to be effective in alleviating medical students' stress and combating burnout problems.

Our study shows that although empathy did not have a main effect on life satisfaction, it interacted with burnout and exerted an interaction effect on the levels of medical students' life satisfaction. Medical students with high levels of empathy and low levels of burnout enjoyed life most, and this finding sheds light on identifying feasible interventions to enhance students' empathy and reduce burnout problems to improve their life satisfaction. It is noteworthy that the effect size was larger for the mean difference in life satisfaction in high empathy groups with low and high burnout levels than in low empathy groups with low and high burnout levels $\left(\eta_{\mathrm{p}}{ }^{2}=\right.$ 0.176 vs $\eta_{\mathrm{p}}{ }^{2}=0.056$ ). This finding indicates that students with high empathy were probably more affected by burnout with regard to life satisfaction than students with low empathy, which lends further support to the idea that enhancing students' empathy and reducing burnout need to be simultaneously addressed to meet the target of achieving high life satisfaction levels. Unlike among health professionals, empathy may not exert such a significant impact on burnout among undergraduate medical students, especially among preclinical students who have little or no contact with real patients. However, cultivating students' empathy is critical and needs to be emphasized from the outset of medical education as this has a long-term effect. At present, some commonly known measures to enhance empathy include early exposure to clinical training, inter-professional education (IPE), a curriculum emphasizing communication and interpersonal skills, a self-reflection approach, creating a culture of humanism and teachers as role models in medical education. Since studies show that the empathy levels of medical students may erode as they step into higher grades, there is an urgent need to experiment with different methods to enhance empathy and implement effective methods in medical education.

Several limitations of the present study should be acknowledged. First, with the cross-sectional design, conclusions about causality cannot be drawn, and longitudinal studies should be carried out in the future. Second, as self-administered questionnaires were distributed to collect data, response bias and social desirability bias cannot be avoided. Third, the present study was conducted in one medical university in China, so generalizations of the conclusions should be made with caution and future multi-institutional research in different 
cultures is suggested. Fourth, the present study analysed the effect of empathy on burnout and life satisfaction of medical students, but in addition to empathy, other variables such as self-efficacy and self-esteem also influenced burnout and life satisfaction levels in medical students, which should be examined in future studies.

\section{Conclusions}

This study explored the relationship among empathy, burnout and life satisfaction and the associated sociodemographic factors in Chinese undergraduate medical students. The results show that the levels of empathy were related to students' age and grade, and levels of burnout were associated with students' maternal education. As for life satisfaction, significant differences were detected with regard to medical students' age, academic year, the number of children in the family, place of residence and parents' educational levels. Over the four academic years, medical students' empathy levels saw a decline, but their burnout levels almost plateaued, and their life satisfaction levels witnessed an initial fall before a rebound. There was an interaction effect of empathy and burnout on life satisfaction, and students with high empathy and low burnout were most satisfied with life. Medical institutions and related authorities need to find effective measures to enhance students' empathy levels and reduce burnout problems to improve their life satisfaction.

\section{Supplementary information}

Supplementary information accompanies this paper at https://doi.org/10. 1186/s12909-019-1788-3.

Additional file 1: Data on main effects and simple effects of empathy and burnout on life satisfaction by ANOVA post hoc Boferroni tests. (DOCX $58 \mathrm{~kb}$ )

\section{Abbreviations \\ EC: Empathic Concern; ESWIM: Empathy, Spirituality, and Wellness in Medicine survey; FS: Fantasy Scale; IB: Improper Behaviour; IPE: Inter- professional education; IRI: Interpersonal Reactivity Index; IRI-C: Interpersonal Reactivity Index Chinese version; JSE: Jefferson Scale of Empathy; JSPE- S: student version of the Jefferson Scale of Physician Empathy; LA: Low Achievement; LM: Low Mood; MBI: Maslach Burnout Inventory; MBI- MC: Maslach Burnout Inventory Modified Chinese version; MBI-SS: Maslach Burnout Inventory-Student Survey; OLBI: Oldenburg Burnout Inventory; PD: Personal Distress; PT: Perspective Taking; SEM: structural equation modelling; SWLS: Satisfaction With Life Scale}

\section{Acknowledgements}

The authors would like to thank the faculty who organized the distribution and collection of the questionnaires in their classrooms as well as the students for their participation.

\section{Authors' contributions}

QHW was in charge of the study design, questionnaire survey, drafting and revising the manuscript. LW and MS contributed to the study design and provided some valuable advice. $\mathrm{XLL}, \mathrm{RL}, \mathrm{JL}$ and $\mathrm{MZ}$ helped to collect and analyze the data. HZW made substantial intellectual contributions to the conception of the study, analyses of the data and draft of the manuscript. All authors read and approved the final version of the manuscript.

\section{Funding}

None declared.

\section{Availability of data and materials}

The datasets used and/or analyzed in the present study are available from the corresponding author on reasonable request.

Ethics approval and consent to participate

All subjects provided written informed consents and the study procedures were approved by the Committee on Human Experimentation of China Medical University.

\section{Consent for publication}

Not applicable.

\section{Competing interests}

The authors declare that they have no competing interests.

\section{Author details}

${ }^{1}$ English Department, School of Fundamental Sciences, China Medical University, No. 77 Puhe Road, Shenyang North New Area, Shenyang, Liaoning Province, People's Republic of China. ${ }^{2}$ Department of Social Medicine, School of Public Health, China Medical University, No. 77 Puhe Road, Shenyang North New Area, Shenyang, Liaoning Province, People's Republic of China. ${ }^{3}$ Department of Epidemiology, School of Public Health, China Medical University, No. 77 Puhe Road, Shenyang North New Area, Shenyang, Liaoning Province, People's Republic of China. ${ }^{4}$ Department of Statistics, School of Public Health, China Medical University, No. 77 Puhe Road, Shenyang North New Area, Shenyang, Liaoning Province, People's Republic of China. ${ }^{5}$ Department of Healthcare Management, School of Humanities and Social Sciences, China Medical University, No. 77 Puhe Road, Shenyang North New Area, Shenyang, Liaoning Province, People's Republic of China.

Received: 17 June 2019 Accepted: 3 September 2019

Published online: 06 September 2019

\section{References}

1. Yuguero O, Melnick ER, Marsal JR, Esquerda M, Soler-Gonzalez J. Crosssectional study of the association between healthcare professionals' empathy and burnout and the number of annual primary care visits per patient under their care in Spain. BMJ Open. 2018:8:e020949.

2. Yuguero O, Marsal JR, Buti M, Esquerda M, Soler-González J. Descriptive study of association between quality of care and empathy and burnout in primary care. BMC Med Ethics. 2017;18:54-61.

3. Duarte J, Pinto-Gouveia J. Empathy and feelings of guilt experienced by nurses: a cross-sectional study of their role in burnout and compassion fatique symptoms. Appl Nurs Res. 2017;35:42-7.

4. Silver J, Caleshu C, Casson-Parkin S, Ormond K. Mindfulness among genetic counselors is associated with increased empathy and work engagement and decreased burnout and compassion fatigue. J Genet Couns. 2018;27: 1175-86.

5. Hojat M. Empathy in patient care: antecedents, development, measurement, and outcomes. New York: Springer; 2007.

6. Kim SS, Kaplowitz S, Johnston MV. The effects of physician empathy on patient satisfaction and compliance. Eval Health Prof. 2004;27(3):237-51.

7. Hojat M, Spandofer J, Louis DZ, Gonnella JS. Empathic and sympathetic orientations toward patient care: conceptualization, measurement, and psychometrics. Acad Med. 2011;86(8):989-95.

8. Shapiro J. Walking a mile in their patients' shoes: empathy and othering in medical students' education. Philos Ethics Humanit Med. 2008:3:10-20.

9. Maslach C, Jackson SE, Leiter MP. The Maslach burnout inventory. 3rd ed. Palo Alto, CA: Consulting Psychologists Press; 1996.

10. Mazurkiewicz R, Korenstein D, Fallar R, Ripp J. The prevalence and correlations of medical student burnout in the pre-clinical years: a crosssectional study. Psychol Health Med. 2012;17(2):188-95.

11. Liu HC, Yansane Al, Zhang YR, Fu HJ, Hong NR, Kalenderian E. Burnout and study engagement among medical students at Sun Yat-sen University, China: A cross-sectional study. Medicine. 2018;97(15):e0326. 
12. PENŠEK L, SELIČ P. Empathy and burnout in Slovenian family medicine doctors: the first presentation of Jefferson scale of empathy results. Zdr Varst. 2018:57(3):155-65.

13. Lee PT, Loh J, Sng G, Tung J, Yeo KK. Empathy and burnout: a study on residents from a Singapore institution. Singap Med J. 2018;59(1):50-4.

14. Wilkinsona $\mathrm{H}$, Whittington $\mathrm{R}$, Perry $\mathrm{L}$, Eames $\mathrm{C}$. Examining the relationship between burnout and empathy in healthcare professionals: a systematic review. Burn Res. 2017;6:18-29.

15. Yuguero O, Marsal JR, Esquerda M, Vivanco L, Soler-Gonzalez J. Association between low empathy and high burnout among primary care physicians and nurses in Lleida. Spain Eur J Gen Pract. 2017;23(1):4-10.

16. Paro HBMS, Silveira PSP, Perotta B, Gannam S, Enns SC, Giaxa RRB. Empathy among medical students: is there a relation with quality of life and burnout? PLoS One. 2014;9(4):e94133.

17. Hojat M, Vergare M, Isenberg G, Cohen M, Spandorfer J. Underlying construct of empathy, optimism, and burnout in medical students. Int J Med Educ. 2015;6:12-6.

18. Lucchetti G, Damiano RF, DiLalla LF, Lucchetti ALG, Moutinho ILD, Ezequie ODS, et al. Cross-cultural differences in mental health, quality of life, empathy, and burnout between US and Brazilian medical students. Acad Psychiatry. 2018;42:62-7.

19. Diener E, Emmons RA, Larsen RJ, Griffin S. The satisfaction with life scale. J Pers Assess. 1985:49:71-5.

20. Cho E, Jeon S. The role of empathy and psychological need satisfaction in pharmacy students' burnout and well-being. BMC Med Educ. 2019;19:43-54

21. Hakanen JJ, Schaufeli WB. Do burnout and work engagement predict depressive symptoms and life satisfaction? A three-wave seven-year prospective study. J Affect Disord. 2012;141:415-24

22. Demerouti $E$, Bakker AB, Nachreiner F, Schaufeli WB. A model of burnout and life satisfaction amongst nurses. J Adv Nurs. 2000;32(2):454-64.

23. Hojat M, Gonnella JS, Nasca TJ, Mangione S, Vergare M, Magee M. Physician empathy: definition, components, measurement, and relationship to gender and specialty. Am J Psychiatry. 2002;159:1563-9.

24. Davis $\mathrm{MH}$. Measuring individual differences in empathy: evidence for multidimensional approach. J Pers Soc Psychol. 1983;44:113-26.

25. Zhang FF, Dong Y, Wang K, Zhan ZY, Xie LF. Reliability and validity of the Chinese version of the interpersonal reactivity index-C. Chinese J Clin Psychol. 2010;18(2):155-7 (in Chinese).

26. Rong X, Sun BH, Huang XZ, Cai MY, Li WJ. Reliabilities and validities of Chinese version of interpersonal reactivity index. Chinese J Clin Psychol. 2010;18(2):158-60 (in Chinese).

27. Lian R, Yang LX, Wu LH. Relationship between professional commitment and learning burnout of undergraduates and scales developing. Acta Psychol Sin. 2005;37(5):632-6 (in Chinese)

28. Shi M, Liu L, Sun X, Wang L. Associations between symptoms of attentiondeficit/ hyperactivity disorder and life satisfaction in medical students: the mediating effect of resilience. BMC Med Educ. 2018;18:164-70.

29. Shi $M$, Wang $X X$, Bian $Y G$, Wang $L$. The mediating role of resilience in the relationship between stress and life satisfaction among Chinese medical students: a cross-sectional study. BMC Med Educ. 2015;15:16-22.

30. Li DJ, Xu HM, Kang MY, Ma SL. Empathy in Chinese eight-year medical program students: differences by school year, educational stage, and future career preference. BMC Med Educ. 2018;18:241-9.

31. Wen DL, Ma XD, Li HH, Liu ZF, Xian BS, Liu Y. Empathy in Chinese medical students: psychometric characteristics and differences by gender and year of medical education. BMC Med Educ. 2013;13:130-5.

32. Santos MA, Grosseman S, Morelli TC, Giuliano ICB, Erdmann TR. Empathy differences by gender and specialty preference in medical students: a study in Brazil. Int J Med Educ. 2016;7:149-53.

33. Rezayat AA, Shahini NS, Asl HT, Jarahi L, Behdani F, Shojaei SRH, et al. Empathy score among medical students in Mashhad, Iran: study of the Jefferson scale of physician empathy. Electron Physician. 2018;10(7):7101-6.

34. Chatterjee A, Ravikumar R, Singh S, Chauhan PS, Goel M. Clinical empathy in medical students in India measured using the Jefferson scale of empathystudent version. J Educ Eval Health Prof. 2017;14:33-8.

35. Conant LL, Liebenthal E, Desai A, Binder JR. The relationship between maternal education and the neural substrates of phoneme perception in children: interactions between socioeconomic status and proficiency level. Brain Lang. 2017;171:14-22.

36. Washi S, Cowan D, Terry RD. The impact of mother's education on indicators of school performance of first through third grade primary school children living in low socioeconomic areas in Khartoum. Sudan Ahfad J. 1993:10(1):44-55.

37. Carvalho C, Cruz O. Disciplinary behavior of mothers of preschool children: effects of maternal efficacy beliefs, children's gender and age, and mothers' education. Estudos de Psicologia (Campinas). 2018;35(4):433-43.

38. Park KH, Kim DH, Kim SK, Yi YH, Jeong JH, Chae J, et al. The relationships between empathy, stress and social support among medical students. Int J Med Educ. 2015;6:103-8.

39. Shi M, Liu L, Wang ZY, Wang L. Prevalence of depressive symptoms and its correlations with positive psychological variables among Chinese medical students: an exploratory cross-sectional study. BMC Psychiatry. 2016;16:3-10.

40. Shi M, Liu L, Yang YL, Wang L. The mediating role of self-esteem in the relationship between big five personality traits and depressive symptoms among Chinese undergraduate medical students. Personal Individ Differ. 2015;83:55-9.

41. Slavin SJ, Schindler DL, Chibnall JT. Medical student mental health 3.0: improving student wellness through curricular changes. Acad Med. 2014; 89(4):573-7.

42. Dyrbye LN, Thomas MR, Harper W, Massie FS Jr, Power DV, Eacker A, et al. The learning environment and medical student burnout: a multicentre study. Med Educ. 2009;43:274-82.

43. Erschens R, Lodaa T, Herrmann-Wernera A, Keifenheima KE, Stuber F, Nikendei $C$, et al. Behaviour-based functional and dysfunctional strategies of medical students to cope with burnout. Med Educ Online. 2018;23:1535738.

44. Galam E, Soupault CV, Bunge L, du Vaure CB, Boujut E, Jaury P. 'Intern life': a longitudinal study of burnout, empathy, and coping strategies used by French GPs in training. BJGP Open. 2017. https://doi.org/10.3399/ bjgpopen17X100773.

\section{Publisher's Note}

Springer Nature remains neutral with regard to jurisdictional claims in published maps and institutional affiliations.

Ready to submit your research? Choose BMC and benefit from:

- fast, convenient online submission

- thorough peer review by experienced researchers in your field

- rapid publication on acceptance

- support for research data, including large and complex data types

- gold Open Access which fosters wider collaboration and increased citations

- maximum visibility for your research: over $100 \mathrm{M}$ website views per year

At BMC, research is always in progress.

Learn more biomedcentral.com/submissions 